\title{
NIVELES DE HEMOGLOBINA Y ANEMIA EN GESTANTES ADOLESCENTES ATENDIDAS EN ESTABLECIMIENTOS DEL MINISTERIO DE SALUD DEL PERÚ, 2009-2012
}

\author{
Oscar Munares-García1,2,a, Guillermo Gómez-Guizado1,b
}

\begin{abstract}
RESUMEN
Objetivos. Determinar los niveles de hemoglobina y anemia en gestantes adolescentes atendidas en los establecimientos del Ministerio de Salud del Perú durante los años 2009 y 2012. Materiales y métodos. Estudio transversal de análisis de datos secundarios utilizando el Sistema de Información del Estado Nutricional (SIEN) del niño y la Gestante. Se revisaron 265788 registros de gestantes de 10 a 19 años. Se midieron los niveles de hemoglobina (g/dL) y el porcentaje de anemia en el primer, segundo y tercer trimestre. Se aplicó estadísticas descriptivas con intervalos de confianza al 95\%. Resultados. El 3,4\% de las gestantes tenían entre 10 a 14 años (adolescencia temprana), el 21,6\% entre 15 a 16 años (adolescencia intermedia) y el $75 \%$ entre 17 a 19 años (adolescencia tardía). Los niveles de hemoglobina en las gestantes adolescentes fueron de $11,6 \pm 1,3 \mathrm{~g} / \mathrm{dL}$ para el 2009 y de 11,5 $\pm 1,3 \mathrm{~g} / \mathrm{dL}$ durante los años $2010,2011 \mathrm{y}$ 2012, la frecuencia global de anemia para el 2009 fue de 25,1\% (IC 95\%: 24,4-25,8); para el 2010 de $26,0 \%$ (IC $95 \%$ : $25,3-26,6$ ), para el 2011 de $26,4 \%$ (IC 95\%: 25,8-27,1) y para el 2012 de 25,2\% (IC 95\%: 24,6-25,9). Conclusiones. Los niveles de hemoglobina son en promedio menores para las gestantes residentes en zonas alto andinas. Alrededor de un cuarto de las gestantes adolescentes presentan anemia.
\end{abstract}

Palabras clave: Anemia; Adolescente; Hemoglobinas; Gestación (fuente: DeCS/BIREME).

\section{LEVELS OF ANEMIA AND HEMOGLOBIN IN PREGNANT ADOLESCENTS WHO ATTENDED HEALTH CARE FACILITIES OF MINISTRY OF HEALTH OF PERU, 2009-2012}

\begin{abstract}
Objectives. To determine hemoglobin levels and anemia in pregnant adolescents who attended the health care facilities of the Ministry of Health of Peru between the years 2009 and 2012. Materials and methods. Cross-sectional study of secondary data analysis using the Information System of the Nutritional Status of Children and Pregnant Women (SIEN). 265,788 records of pregnant women aged 10 to 19 years were reviewed. Hemoglobin levels $(\mathrm{g} / \mathrm{dL})$ and the percentage of anemia in the first, second and third trimesters were measured. Descriptive statistics with confidence intervals at $95 \%$ were applied. Results. 3.4\% of pregnant women were aged 10 to 14 years (early adolescence), $21.6 \%$ between 15 to 16 years (middle adolescence) and $75 \%$ between 17 to 19 years (late adolescence). Hemoglobin levels in pregnant adolescents were $11.6 \pm 1.3 \mathrm{~g} / \mathrm{dL}$ in 2009 and $11.5 \pm 1.3 \mathrm{~g} / \mathrm{dL}$ during the years 2010,2011 and 2012 . The overall incidence of anemia for 2009 was $25.1 \%$ (95\% Cl 24.4-25.8); for 2010 was $26.0 \%$ (95\% Cl 25.3-26.6) for 2011 was $26.4 \%$ (95\% Cl 25.8-27.1) and 25.2\% for 2012 (95\% Cl 24.6-25.9). Conclusions. Hemoglobin levels were on average lower for pregnant residents in high Andean areas. About a quarter of pregnant adolescents in our sample had anemia.
\end{abstract}

Key words: Anemia; Adolescent; Hemoglobins; Pregnancy (source: MeSH/NLM).

\footnotetext{
Centro Nacional de Alimentación y Nutrición, Instituto Nacional de Salud. Lima, Perú.

Facultad de Medicina, Universidad Nacional Mayor de San Marcos. Lima, Perú.

Licenciado en Obstetricia, magíster en Salud Pública; ${ }^{\mathrm{b}}$ médico cirujano, especialista en Epidemiologia de Campo.

Parte de esta información fue presentada en el VII Congreso Científico Internacional del Instituto Nacional de Salud 2013.

Recibido: 11-12-13 Aprobado: 28-05-14
}

Citar como: Munares-García O, Gómez-Guizado G. Niveles de hemoglobina y anemia en gestantes adolescentes atendidas en establecimientos del Ministerio de Salud del Perú, 2009-2012. Rev Peru Med Exp Salud Publica. 2014;31(3):501-8. 


\section{INTRODUCCIÓN}

La adolescencia, es una etapa de la vida entre los 10 a 19 años, y se calcula que para el 2025 llegará hasta los 2000 millones de habitantes ${ }^{(1)}$. El embarazo en la adolescencia es considerado como un problema de salud pública. Aproximadamente 15 millones dan a luz cada año y, de los 13 millones de partos en América Latina y el Caribe, dos millones, aproximadamente un $15 \%$, son de adolescentes ${ }^{(2)}$. Según la Encuesta Demográfica y de Salud Familiar del año 2009, el porcentaje de madres del total de adolescentes entre los 15 a 19 años fue del $13,7 \%$ y para el 2012 fue del $13,2 \%{ }^{(3)}$. La familia de una adolescente embarazada atraviesa una serie de sentimientos y transformaciones en su dinámica, generando una problemática familiar que muchas veces repercute en las relaciones familiares comprometiendo la salud de la adolescente y el recién nacido ${ }^{(4)}$.

El embarazo adolescente trae consigo problemas como el mayor riesgo de anemia ${ }^{(5)}$. Según la Organización Mundial de la Salud (OMS), en el año 2005 la anemia afectó a 1620 millones de personas en el mundo, cerca del $24,8 \%$ de la población mundial, donde los grupos más afectados son los niños en edad preescolar $(47,4 \%)$ y gestantes $(41,8 \%){ }^{(6)}$. La anemia en el embarazo incrementa la morbilidad materna y feto-neonatal siendo considerado como un problema de salud pública en los países en vías de desarrollo ${ }^{(7)}$.

Un estudio previo realizado en el Perú, en el año 2011, determinó que la anemia en gestantes entre los 10 a 15 años fue del $30,7 \%{ }^{(8)}$, pero este dato no fue seguido en un periodo por lo que el objetivo de la presente investigación fue determinar los niveles de hemoglobina y anemia en gestantes adolescentes atendidas en los establecimientos del Ministerio de Salud del Perú en el periodo 2009 a 2012.

\section{MATERIALES Y MÉTODOS}

\section{DISEÑO DEL ESTUDIO Y POBLACIÓN}

Estudio transversal, descriptivo, de análisis de base de datos secundarios, usando el Sistema de Información del Estado Nutricional del Niño (SIEN) y la Gestante, del Centro Nacional de Alimentación y Nutrición (CENAN) del Instituto Nacional de Salud (INS). La población correspondió a 265788 registros de gestantes de 10 a 19 años procedentes de 6530 establecimientos de salud del Ministerio de Salud del Perú entre los años 2009 a 2012; se excluyó 1066 registros por estar incompletos o con inconsistencias.

\section{VARIABLES DE ESTUDIO}

Se midió la edad de la adolescente y se agrupó por etapas de la adolescencia en: temprana (10 a 13 años); intermedia (14 a 16 años), y tardía (17 a 19 años); edad gestacional por trimestre: primero (1-14 semanas); segundo (15-28 semanas), y tercero (29-41 semanas 6 días); gestación (única o múltiple); altitud a nivel del mar; establecimientos de salud y las 25 regiones del Perú. La hemoglobina se midió en $\mathrm{g} / \mathrm{dL}$ correspondiente al último control prenatal registrado en el aplicativo. Se determinó anemia para la edad si el resultado de $\mathrm{Hb}<11 \mathrm{~g} / \mathrm{dL}^{(9)}$ entre el primer y tercer trimestre y $<10,5 \mathrm{~g} / \mathrm{dL}$ para el segundo trimestre ${ }^{(10)}$. De acuerdo a lo indicado por la OMS ${ }^{(11)}$ se clasificó en anemia leve $(\mathrm{Hb}$ de 10-10,9 g/dL); moderada (Hb de 7-9,9 g/dL) y severa $(\mathrm{Hb}<7 \mathrm{~g} / \mathrm{dL})$. Los valores de hemoglobina fueron ajustados a la altitud a nivel del mar de acuerdo a norma OMS en el aplicativo SIEN.

\section{ANÁLISIS ESTADÍSTICO}

Se aplicó distribución de frecuencias y porcentajes para las variables adolescencia, trimestre de embarazo, tipo de embarazo, altitud sobre el nivel del mar, establecimiento de salud y región. Así mismo, se aplicaron promedios y desviaciones estándar e intervalos de confianza al 95\% para los niveles de hemoglobina de acuerdo a las variables indicadas, por años, y se determinó la proporción de anemia con sus intervalos de confianza al $95 \%$.

\section{CONSIDERACIONES ÉTICAS}

La base de datos no contó con nombres, ni datos de identificación de las gestantes, salvo el número de historia clínica de cada centro de salud, que fue obviado del análisis, por lo que los datos del análisis fueron anónimos.

\section{RESULTADOS}

El 75\% (199 336/265 788) de las gestantes se encontraban entre los 17 a 19 años de edad; el 92,7\% entre el segundo y tercer trimestre. El 99,7\% presentó gestación única. El 59,6\% residía a una altitud menor a 1000 metros. El $91,2 \%$ fueron atendidas en puestos o centros de salud y el $31,9 \%$ provenían de las regiones de Lima, Huánuco y Cajamarca (Tabla 1).

Para el año 2009, el promedio de $\mathrm{Hb}$ fue de 11,6 \pm $1,3 \mathrm{~g} / \mathrm{dL}$ y para el año 2010 a 2012 de $11,5 \pm 1,3 \mathrm{~g} / \mathrm{dL}$. El promedio de $\mathrm{Hb}$ fue mayor en el primer trimestre de gestación (12,0 $\pm 1,2 \mathrm{~g} / \mathrm{dL})$, esta característica 
Tabla 1. Distribucion de las gestantes adolescentes atendidas en establecimientos del Ministerio de Salud, Perú 2009-2012

\begin{tabular}{|c|c|c|}
\hline & $\mathbf{N}$ & $(\%)$ \\
\hline \multicolumn{3}{|l|}{ Adolescencia } \\
\hline Temprana & 9141 & $(3,4)$ \\
\hline Intermedia & 57311 & $(21,6)$ \\
\hline Tardía & 199336 & $(75,0)$ \\
\hline \multicolumn{3}{|l|}{ Trimestre } \\
\hline Primero & 19225 & $(7,2)$ \\
\hline Segundo & 89936 & $(33,8)$ \\
\hline Tercero & 156627 & $(58,9)$ \\
\hline \multicolumn{3}{|l|}{ Gestación } \\
\hline Única & 265034 & $(99,7)$ \\
\hline Múltiple & 754 & $(0,3)$ \\
\hline \multicolumn{3}{|l|}{ Altitud } \\
\hline 2 a 1000 & 158485 & $(59,6)$ \\
\hline $1001-2000$ & 18208 & $(6,9)$ \\
\hline $2001-3000$ & 36380 & $(13,7)$ \\
\hline $3001-4000$ & 49016 & $(18,4)$ \\
\hline $4001-4801$ & 3699 & $(1,4)$ \\
\hline \multicolumn{3}{|c|}{ Establecimiento de salud } \\
\hline Puesto de salud & 114357 & $(43,0)$ \\
\hline Centro de salud & 128001 & $(48,2)$ \\
\hline Hospital & 23430 & $(8,8)$ \\
\hline \multicolumn{3}{|l|}{ Región } \\
\hline Amazonas & 4396 & $(1,7)$ \\
\hline Ancash & 11233 & $(4,2)$ \\
\hline Apurímac & 5069 & $(1,9)$ \\
\hline Arequipa & 8323 & $(3,1)$ \\
\hline Ayacucho & 12300 & $(4,6)$ \\
\hline Cajamarca & 16618 & $(6,3)$ \\
\hline Callao & 6011 & $(2,3)$ \\
\hline Cusco & 15269 & $(5,7)$ \\
\hline Huancavelica & 5950 & $(2,2)$ \\
\hline Huánuco & 17657 & $(6,6)$ \\
\hline Ica & 7598 & $(2,9)$ \\
\hline Junín & 12563 & $(4,7)$ \\
\hline La Libertad & 16256 & $(6,1)$ \\
\hline Lambayeque & 10434 & $(3,9)$ \\
\hline Lima & 50541 & $(19,0)$ \\
\hline Loreto & 14324 & $(5,4)$ \\
\hline Madre de Dios & 2145 & $(0,8)$ \\
\hline Moquegua & 952 & $(0,4)$ \\
\hline Pasco & 4260 & $(1,6)$ \\
\hline Piura & 10019 & $(3,8)$ \\
\hline Puno & 9149 & $(3,4)$ \\
\hline San Martín & 13541 & $(5,1)$ \\
\hline Tacna & 1653 & $(0,6)$ \\
\hline Tumbes & 3541 & $(1,3)$ \\
\hline Ucayali & 5986 & $(2,3)$ \\
\hline Total & 265788 & $(100)$ \\
\hline
\end{tabular}

se mantuvo en los años evaluados. El promedio de $\mathrm{Hb}$ fue menor conforme aumenta la altitud a nivel del mar. El promedio de $\mathrm{Hb}$ fue similar según el tipo de gestación única o multiple. La región de Huancavelica mantuvo los promedios más bajos de $\mathrm{Hb}$ durante los años de estudio. La región de Lambayeque presentó el mayor promedio de $\mathrm{Hb}$ durante los años 2009 a 2012 (Tabla 2).

Menos del $30 \%$ de las gestantes presentaron anemia, este comportamiento se mantuvo a lo largo del periodo. La frecuencia de anemia fue menor en la adolescencia temprana con porcentajes entre 21,5 a $25,7 \%$ en el periodo. Para el año 2012 el 15,8\% presentó anemia en el primer trimestre, 16,6\% para el segundo trimestre y de $32,5 \%$ para el tercer trimestre, la proporción entre gestaciones únicas y múltiples fue similar. Con respecto a la altitud a nivel del mar, a mayor altitud, mayor es la frecuencia de anemia, este comportamiento se observó en todos los años, siendo las frecuencias más altas para las gestantes residentes a más de 4000 metros. Cuatro regiones presentaron las cifras más altas de anemia, para el año 2009: Apurímac, Huancavelica y Puno; para el año 2010, Apurímac, Ayacucho, Huancavelica y Puno; para el año 2011, Ayacucho, Huancavelica y Puno, y para el año 2012: Ayacucho, Huancavelica y Puno (Tabla 3).

De acuerdo con la edad, los porcentajes de anemia son más heterogeneas, entre los 10 a 14 años, y tienden a ser más consistentes desde los 15 a 19 años, manteniendo proporciones de anemia por encima del $24 \%$ (Figura 1). La frecuencia global de anemia en la gestante adolescente se encontró entre $25,1 \%$ en el año 2009 a 26,4\% en el 2011. La anemia leve presentó frecuencias por encima del 15,4\% (Tabla 4).

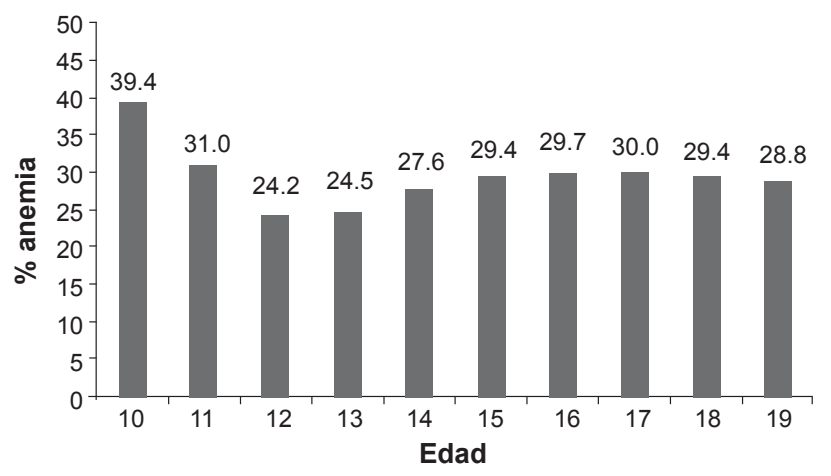

Figura 1. Distribución porcentual del promedio de anemia en gestantes adolescentes atendidas en el Ministerio de Salud, Perú, 2009-2012. 
Tabla 2. Niveles de hemoglobina $(\mathrm{g} / \mathrm{dL})$ de gestantes adolescentes atendidas en establecimientos del Ministerio de Salud, Perú 2009-2012

\begin{tabular}{|c|c|c|c|c|c|c|c|c|}
\hline & \multicolumn{2}{|c|}{2009} & \multicolumn{2}{|c|}{2010} & \multicolumn{2}{|c|}{2011} & \multicolumn{2}{|r|}{2012} \\
\hline & $\mathbf{N}$ & Media (DE) & $\mathbf{N}$ & Media (DE) & $\mathbf{N}$ & Media (DE) & $\mathbf{N}$ & Media (DE) \\
\hline \multicolumn{9}{|l|}{ Adolescencia } \\
\hline Temprana & 1991 & $11,6(1,3)$ & 2282 & $11,6(1,2)$ & 2401 & $11,5(1,3)$ & 2467 & $11,5(1,3)$ \\
\hline Intermedia & 13376 & $11,5(1,3)$ & 14608 & $11,5(1,3)$ & 14761 & $11,5(1,3)$ & 14566 & $11,5(1,3)$ \\
\hline Tardía & 44329 & $11,6(1,3)$ & 50001 & $11,5(1,3)$ & 51570 & $11,5(1,3)$ & 53436 & $11,5(1,3)$ \\
\hline \multicolumn{9}{|l|}{ Trimestre } \\
\hline Primero & 3721 & $12,0(1,2)$ & 4426 & $12,0(1,2)$ & 5099 & $12,0(1,3)$ & 5979 & $12,0(1,2)$ \\
\hline Segundo & 18834 & $11,6(1,3)$ & 21723 & $11,6(1,3)$ & 23437 & $11,6(1,3)$ & 25942 & $11,6(1,3)$ \\
\hline Tercero & 37141 & $11,5(1,3)$ & 40742 & $11,5(1,3)$ & 40196 & $11,4(1,3)$ & 38548 & $11,4(1,3)$ \\
\hline \multicolumn{9}{|l|}{ Gestación } \\
\hline Única & 59510 & $11,6(1,3)$ & 66772 & $11,5(1,3)$ & 68519 & $11,5(1,3)$ & 70233 & $11,5(1,3)$ \\
\hline Múltiple & 186 & $11,6(1,2)$ & 119 & $11,5(1,5)$ & 213 & $11,5(1,3)$ & 236 & $11,5(1,1)$ \\
\hline \multicolumn{9}{|l|}{ Altitud } \\
\hline $2-1000$ & 35797 & $11,6(1,2)$ & 39511 & $11,7(1,2)$ & 40472 & $11,7(1,2)$ & 42705 & $11,7(1,1)$ \\
\hline $1001-2000$ & 3979 & $11,8(1,2)$ & 4900 & $11,7(1,2)$ & 4804 & $11,7(1,2)$ & 4525 & $11,7(1,2)$ \\
\hline $2001-3000$ & 8782 & $11,8(1,3)$ & 8967 & $11,7(1,3)$ & 9278 & $11,6(1,4)$ & 9353 & $11,6(1,3)$ \\
\hline $3001-4000$ & 10400 & $11,1(1,4)$ & 12465 & $11,0(1,4)$ & 13166 & $10,9(1,5)$ & 12985 & $10,9(1,5)$ \\
\hline $4001-4801$ & 738 & $10,9(1,7)$ & 1048 & $10,9(1,7)$ & 1012 & $10,7(1,5)$ & 901 & $10,8(1,6)$ \\
\hline \multicolumn{9}{|l|}{ Región } \\
\hline Amazonas & 862 & $11,7(1,3)$ & 955 & $11,8(1,4)$ & 1241 & $11,9(1,2)$ & 1338 & $11,9(1,1)$ \\
\hline Ancash & 2520 & $11,4(1,3)$ & 2938 & $11,4(1,3)$ & 2964 & $11,4(1,3)$ & 2811 & $11,4(1,3)$ \\
\hline Apurímac & 1161 & $11,1(1,8)$ & 1153 & $11,2(1,8)$ & 1291 & $11,1(1,6)$ & 1464 & $11,3(1,4)$ \\
\hline Arequipa & 2052 & $11,9(1,2)$ & 2035 & $11,9(1,2)$ & 2105 & $11,9(1,2)$ & 2131 & $11,9(1,4)$ \\
\hline Ayacucho & 2932 & $11,3(1,4)$ & 3029 & $11,2(1,4)$ & 3200 & $11,0(1,5)$ & 3139 & $10,9(1,4)$ \\
\hline Cajamarca & 3853 & $11,9(1,1)$ & 4351 & $11,9(1,2)$ & 4094 & $11,7(1,2)$ & 4320 & $11,6(1,3)$ \\
\hline Callao & 1128 & $11,1(1,0)$ & 1491 & $11,2(1,2)$ & 1693 & $11,4(1,1)$ & 1699 & $11,4(1,1)$ \\
\hline Cusco & 3110 & $11,6(1,5)$ & 3873 & $11,4(1,5)$ & 4221 & $11,3(1,5)$ & 4065 & $11,2(1,5)$ \\
\hline Huancavelica & 952 & $10,8(1,7)$ & 1791 & $10,7(1,5)$ & 1708 & $10,7(1,6)$ & 1499 & $11,0(1,7)$ \\
\hline Huánuco & 4310 & $11,7(1,4)$ & 4478 & $11,4(1,3)$ & 4452 & $11,5(1,3)$ & 4417 & $11,5(1,3)$ \\
\hline Ica & 1772 & $11,7(1,1)$ & 1746 & $11,8(1,2)$ & 2018 & $11,8(1,2)$ & 2062 & $11,7(1,2)$ \\
\hline Junín & 2762 & $11,6(1,2)$ & 3085 & $11,4(1,3)$ & 3484 & $11,4(1,4)$ & 3232 & $11,3(1,4)$ \\
\hline La Libertad & 4266 & $11,6(1,1)$ & 3958 & $11,5(1,2)$ & 3697 & $11,5(1,2)$ & 4335 & $11,5(1,2)$ \\
\hline Lambayeque & 2667 & $12,0(1,1)$ & 2835 & $12,0(1,2)$ & 2354 & $12,0(1,1)$ & 2578 & $11,9(1,1)$ \\
\hline Lima & 10864 & $11,5(1,1)$ & 12541 & $11,5(1,1)$ & 13268 & $11,5(1,1)$ & 13868 & $11,6(1,1)$ \\
\hline Loreto & 3202 & $11,5(1,2)$ & 3024 & $11,7(1,1)$ & 3435 & $11,7(1,1)$ & 4663 & $11,7(1,1)$ \\
\hline $\begin{array}{l}\text { Madre de } \\
\text { Dios }\end{array}$ & 435 & $11,8(1,5)$ & 675 & $11,8(1,2)$ & 559 & $11,4(1,4)$ & 476 & $11,4(1,3)$ \\
\hline Moquegua & 174 & $11,6(1,1)$ & 291 & $11,4(1,5)$ & 171 & $11,7(1,3)$ & 316 & $11,6(1,3)$ \\
\hline Pasco & 1037 & $11,3(1,5)$ & 1150 & $11,3(1,5)$ & 1144 & $11,2(1,4)$ & 929 & $11,3(1,4)$ \\
\hline Piura & 2209 & $11,7(1,1)$ & 2812 & $11,8(1,1)$ & 2565 & $11,7(1,1)$ & 2433 & $11,8(1,1)$ \\
\hline Puno & 1809 & $11,0(1,3)$ & 2233 & $11,0(1,4)$ & 2546 & $10,9(1,3)$ & 2561 & $10,9(1,3)$ \\
\hline San Martín & 3519 & $11,8(1,1)$ & 3791 & $11,7(1,1)$ & 3130 & $11,6(1,2)$ & 3101 & $11,8(1,1)$ \\
\hline Tacna & 422 & $11,7(1,2)$ & 407 & $11,7(1,3)$ & 396 & $11,7(1,3)$ & 428 & $11,7(1,3)$ \\
\hline Tumbes & 669 & $11,8(1,2)$ & 795 & $11,8(1,1)$ & 990 & $11,8(1,2)$ & 1087 & $11,7(1,2)$ \\
\hline Ucayali & 1009 & $11,5(1,3)$ & 1454 & $11,6(1,2)$ & 2006 & $11,7(1,2)$ & 1517 & $11,6(1,2)$ \\
\hline Total & 59696 & $11,6(1,3)$ & 66891 & $11,5(1,3)$ & 68732 & $11,5(1,3)$ & 70469 & $11,5(1,3)$ \\
\hline
\end{tabular}

DE: Desviación estándar 
Tabla 3. Distribución de gestantes adolescentes con anemia atendidas en establecimientos del Ministerio de Salud, Perú 2009-2012

\begin{tabular}{|c|c|c|c|c|c|c|c|c|}
\hline & \multicolumn{2}{|c|}{2009} & \multicolumn{2}{|r|}{2010} & \multicolumn{2}{|r|}{2011} & \multicolumn{2}{|c|}{2012} \\
\hline & \multicolumn{2}{|c|}{$n=59696$} & \multicolumn{2}{|c|}{$n=66891$} & \multicolumn{2}{|c|}{$n=68732$} & \multicolumn{2}{|c|}{$n=70469$} \\
\hline & $\%$ & (IC 95\%) & $\%$ & (IC 95\%) & $\%$ & (IC 95\%) & $\%$ & (IC 95\%) \\
\hline \multicolumn{9}{|l|}{ Adolescencia } \\
\hline Temprana & 21,5 & $(17,6-25,4)$ & 21,7 & $(18,1-25,3)$ & 25,7 & $(22,2-29,1)$ & 23,3 & $(19,8-26,7)$ \\
\hline Intermedia & 25,1 & $(23,7-26,6)$ & 25,5 & $(24,1-26,9)$ & 26,7 & $(25,4-28,1)$ & 26,0 & $(24,6-27,4)$ \\
\hline Tardía & 25,3 & $(24,5-26,1)$ & 26,3 & $(25,6-27,1)$ & 26,4 & $(25,6-27,1)$ & 25,1 & $(24,4-25,9)$ \\
\hline \multicolumn{9}{|l|}{ Trimestre } \\
\hline Primero & 16,6 & $(13,6-19,5)$ & 16,1 & $(13,4-18,8)$ & 16,9 & $(14,4-19,4)$ & 15,8 & $(13,5-18,1)$ \\
\hline Segundo & 17,0 & $(15,7-18,3)$ & 17,0 & $(15,8-18,2)$ & 17,5 & $(16,3-18,6)$ & 16,6 & $(15,5-17,7)$ \\
\hline Tercero & 30,1 & $(29,3-31,0)$ & 31,9 & $(31,1-32,7)$ & 32,9 & $(32,1-33,7)$ & 32,5 & $(31,7-33,4)$ \\
\hline \multicolumn{9}{|l|}{ Gestación } \\
\hline Única & 25,1 & $(24,4-25,8)$ & 26,0 & $(25,3-26,6)$ & 26,4 & $(25,8-27,1)$ & 25,3 & $(24,6-25,9)$ \\
\hline Múltiple & 26,9 & $(14,6-39,2)$ & 31,1 & $(16,2-46,0)$ & 25,4 & $(13,7-37,0)$ & 22,5 & $(11,2-33,7)$ \\
\hline \multicolumn{9}{|l|}{ Altitud } \\
\hline $2-1000$ & 20,7 & $(19,7-21,6)$ & 19,8 & $(18,9-20,7)$ & 19,9 & $(19,0-20,8)$ & 19,2 & $(18,4-20,1)$ \\
\hline $1001-2000$ & 20,3 & $(17,6-23,1)$ & 21,7 & $(19,2-24,2)$ & 22,1 & $(19,7-24,6)$ & 19,7 & $(17,1-22,3)$ \\
\hline $2001-3000$ & 23,5 & $(21,7-25,3)$ & 25,3 & $(23,5-27,1)$ & 26,4 & $(24,7-28,2)$ & 26,7 & $(25,0-28,5)$ \\
\hline $3001-4000$ & 41,9 & $(40,5-43,4)$ & 45,8 & $(44,5-47,0)$ & 46,0 & $(44,8-47,3)$ & 44,2 & $(43,0-45,5)$ \\
\hline $4001-4801$ & 50,3 & $(45,2-55,4)$ & 49,8 & $(45,5-54,1)$ & 53,3 & $(49,0-57,5)$ & 49,1 & $(44,4-53,7)$ \\
\hline \multicolumn{9}{|l|}{ Región } \\
\hline Amazonas & 22,7 & $(16,9-28,6)$ & 19,0 & $(13,2-24,7)$ & 16,4 & $(11,3-21,4)$ & 15,2 & $(10,3-20,2)$ \\
\hline Ancash & 32,6 & $(29,4-35,8)$ & 27,8 & $(24,7-30,8)$ & 26,7 & $(23,6-29,8)$ & 26,5 & $(23,3-29,7)$ \\
\hline Apurímac & 43,4 & $(39,1-47,7)$ & 41,5 & $(37,1-46,0)$ & 38,8 & $(34,5-43,1)$ & 30,7 & $(26,5-35,0)$ \\
\hline Arequipa & 18,9 & $(15,0-22,8)$ & 18,5 & $(14,6-22,4)$ & 16,1 & $(12,1-20,0)$ & 16,5 & $(12,6-20,4)$ \\
\hline Ayacucho & 39,4 & $(36,5-42,2)$ & 40,7 & $(38,0-43,4)$ & 44,2 & $(41,6-46,7)$ & 47,1 & $(44,5-49,6)$ \\
\hline Cajamarca & 16,7 & $(13,8-19,5)$ & 16,3 & $(13,6-19,0)$ & 22,0 & $(19,3-24,7)$ & 23,3 & $(20,7-25,9)$ \\
\hline Callao & 36,9 & $(32,2-41,5)$ & 34,7 & $(30,6-38,8)$ & 27,5 & $(23,5-31,6)$ & 24,2 & $(20,1-28,4)$ \\
\hline Cusco & 28,8 & $(25,8-31,7)$ & 34,9 & $(32,3-37,4)$ & 34,4 & $(32,0-36,9)$ & 36,4 & $(33,9-38,8)$ \\
\hline Huancavelica & 48,3 & $(43,8-52,9)$ & 53,7 & $(50,5-56,8)$ & 49,9 & $(46,5-53,2)$ & 40,0 & $(36,0-43,9)$ \\
\hline Huánuco & 23,9 & $(21,3-26,5)$ & 28,9 & $(26,4-31,4)$ & 28,4 & $(25,9-30,9)$ & 25,5 & $(23,0-28,1)$ \\
\hline Ica & 18,1 & $(13,9-22,3)$ & 16,2 & $(11,9-20,5)$ & 18,9 & $(15,0-22,8)$ & 18,8 & $(14,9-22,7)$ \\
\hline Junín & 26,2 & $(23,0-29,4)$ & 31,8 & $(28,9-34,7)$ & 32,3 & $(29,6-35,1)$ & 32,9 & $(30,1-35,7)$ \\
\hline La Libertad & 23,5 & $(20,9-26,1)$ & 26,4 & $(23,7-29,0)$ & 27,2 & $(24,4-29,9)$ & 27,9 & $(25,4-30,5)$ \\
\hline Lambayeque & 12,2 & $(8,6-15,7)$ & 12,5 & $(9,1-16,0)$ & 13,8 & $(10,1-17,6)$ & 14,2 & $(10,7-17,8)$ \\
\hline Lima & 24,4 & $(22,7-26,0)$ & 23,9 & $(22,4-25,4)$ & 22,4 & $(20,9-23,9)$ & 20,4 & $(18,9-21,9)$ \\
\hline Loreto & 22,7 & $(19,6-25,7)$ & 15,9 & $(12,6-19,1)$ & 16,7 & $(13,7-19,8)$ & 18,0 & $(15,4-20,6)$ \\
\hline Madre de Dios & 21,1 & $(12,8-29,5)$ & 18,1 & $(11,2-24,9)$ & 27,0 & $(19,9-34,1)$ & 29,4 & $(21,9-37,0)$ \\
\hline Moquegua & 23,6 & $(10,6-36,6)$ & 36,1 & $(26,9-45,3)$ & 22,8 & $(9,6-36,0)$ & 27,2 & $(17,8-36,6)$ \\
\hline Pasco & 34,7 & $(29,8-39,6)$ & 33,9 & $(29,2-38,6)$ & 38,8 & $(34,3-43,3)$ & 35,1 & $(29,9-40,3)$ \\
\hline Piura & 17,4 & $(13,6-21,2)$ & 17,4 & $(14,0-20,7)$ & 20,2 & $(16,7-23,7)$ & 18,1 & $(14,5-21,7)$ \\
\hline Puno & 45,6 & $(42,2-49,0)$ & 46,1 & $(43,1-49,2)$ & 46,1 & $(43,3-49,0)$ & 43,9 & $(41,0-46,8)$ \\
\hline San Martín & 16,4 & $(13,4-19,4)$ & 17,8 & $(14,9-20,6)$ & 21,0 & $(17,8-24,1)$ & 17,4 & $(14,2-20,6)$ \\
\hline Tacna & 20,4 & $(11,9-28,9)$ & 19,7 & $(10,9-28,4)$ & 20,7 & $(11,9-29,5)$ & 19,4 & $(10,9-27,9)$ \\
\hline Tumbes & 19,9 & $(13,1-26,7)$ & 19,6 & $(13,4-25,9)$ & 16,7 & $(11,0-22,4)$ & 18,3 & $(12,9-23,7)$ \\
\hline Ucayali & 24,4 & $(19,0-29,7)$ & 19,8 & $(15,2-24,4)$ & 18,8 & $(14,9-22,7)$ & 20,6 & $(16,1-25,1)$ \\
\hline Total & 25,1 & $(24,4-25,8)$ & 26,0 & $(25,3-26,6)$ & 26,4 & $(25,8-27,1)$ & 25,2 & $(24,6-25,9)$ \\
\hline
\end{tabular}


Tabla 4. Distribución porcentual de los niveles de anemia en gestantes adolescentes atendidas en establecimientos del Ministerio de Salud, Perú, 2009-2012

\begin{tabular}{|c|c|c|c|c|c|c|c|c|}
\hline & \multicolumn{2}{|r|}{2009} & \multicolumn{2}{|r|}{2010} & \multicolumn{2}{|r|}{2011} & \multicolumn{2}{|r|}{2012} \\
\hline & $\%$ & (IC 95\%) & $\%$ & (IC 95\%) & $\%$ & (IC 95\%) & $\%$ & (IC 95\%) \\
\hline Normal & 74,9 & $(74,5-75,3)$ & 74,0 & $(73,6-74,4)$ & 73,6 & $(73,2-74,0)$ & 74,8 & $(74,4-75,1)$ \\
\hline Anemia leve & 16,0 & $(15,3-16,7)$ & 16,1 & $(15,4-16,8)$ & 15,9 & $(15,2-16,6)$ & 15,4 & $(14,8-16,1)$ \\
\hline Anemia moderada & 9,0 & $(8,2-9,7)$ & 9,7 & $(9,0-10,5)$ & 10,3 & $(9,6-11,0)$ & 9,5 & $(8,8-10,2)$ \\
\hline Anemia severa & 0,2 & $(0,0-1,0)$ & 0,2 & $(0,6-0,9)$ & 0,2 & $(0,0-0,9)$ & 0,3 & $(0,0-1,0)$ \\
\hline Anemia global & 25,1 & $(24,4-25,8)$ & 26,0 & $(25,3-26,6)$ & 26,4 & $(25,8-27,1)$ & 25,2 & $(24,6-25,9)$ \\
\hline
\end{tabular}

\section{DISCUSIÓN}

En el Perú, un cuarto de las gestantes adolescentes atendidas en establecimientos del Ministerio de Salud presentan anemia, este evento se ha mantenido durante los años 2009 a 2012. Las cifras presentadas en este estudio se encuentran dentro de los rangos de países latinoamericanos. En México se determinó una frecuencia del 15 a 93\% (12); en Venezuela, para el año 2012 osciló entre 14,9 y 78,0\% (13), y en Colombia la frecuencia de anemia en gestantes adolescentes fue del $15 \%$ para el año $2009{ }^{(14)}$.

Una de las estrategias para reducir la anemia en la gestante es el tratamiento con sulfato ferroso vía oral (15). Para el año 2010, el reporte sobre cumplimiento de metas, tomando como base la información de afiliadas SIS con suplementación con hierro, ubicó a 20 distritos de Apurímac, Ayacucho y Huancavelica con altos porcentajes de cumplimiento ${ }^{(16)}$, por lo que resulta contradictorio que las gestantes adolescentes residentes en estas regiones presenten altas cifras de anemia, ello puede deberse a la falta de supervisión a la adherencia al tratamiento, entre otras causas. Un estudio realizado en gestantes de Apurímac y Ayacucho sobre adherencia al tratamiento con sulfato ferroso, encontró que la proporción de gestantes que tiene un nivel de adherencia óptima fue alrededor del $25 \%$ en las primeras cuatro visitas, para luego caer aproximadamente a $5 \%$ en las últimas ${ }^{(17)}$.

La administración de hierro por vía oral presenta, por lo general, efectos secundarios como náuseas, vómitos, mal sabor de boca y dolor de cabeza, si estos efectos ocurren, la dosis debe ser reducida o se debe usar un producto diferente como el fumarato, succinato o gluconato ferroso, aunque la literatura indica que los efectos secundarios son similares, solo se ha podido demostrar diferencias con el complejo de hierro polimaltosado, pues actúa como una envoltura alrededor del hierro trivalente, asegurando una liberación más lenta ${ }^{(18)}$. Otra alternativa sería la suplementación con micronutrientes múltiples ${ }^{(19)}$, se recomienda considerar otras alternativas que tengan mejor aceptación en las gestantes. Otra herramienta dentro de la estrategia contra la anemia en el embarazo es la consejería nutricional (20) estipuladas también en las Guías de Atención Integral a la Salud Sexual y Reproductiva ${ }^{(21)}$, se indica que el personal que atiende a las gestantes debe ofrecer información oportuna sobre alimentos ricos en hierro de alta biodisponibilidad (sangre, carnes rojas, pescado, vísceras), con una absorción del 15 al $40 \%$, y combinarlos con alimentos con hierro de baja biodisponibilidad (alimentos de origen vegetal, huevos y productos lácteos) ${ }^{(15)}$, siendo necesario garantizar un contínuo reforzamiento al personal que atiende a la gestante para que desarrolle estrategias de mejora de la consejería a nivel local.

Cuatro regionesaltoandinas presentan las cifras másaltas de anemia en gestantes adolescentes, las estrategias requieren de un enfoque más integral desde el punto de vista sociocultural, empezando por la capacitación del personal de salud en esas zonas. La estrategia para manejo de problemas de salud en regiones altoandinas debe de ser reestructurada; un estudio cualitativo hecho en mujeres nicaragüenses demostró que, dependiendo de la etnia donde se originan, presentan creencias diferentes de la concepción y manejo de la anemia ${ }^{(22)}$, es evidente que estas regiones, con creencias culturales diferentes a las occidentales, con alta proporción de pensamiento racional o racionalidad andina, simbólica, afectiva, integradora y de reciprocidad ${ }^{(23)}$, no sea afín a los modos de intervención actuales.

En el Perú, uno de los países de las Américas con mayor proporción de población indígena y diversidad étnica ${ }^{(24)}$, es evidente que el manejo de las estrategias no pueden ser iguales a las que se ofrecen en ciudades como Lima, por ejemplo, Apurímac se encuentra entre 2000 a 4500 metros de altitud, $71,7 \%$ tiene como lengua materna el quechua, aymara o ashaninca u otra lengua nativa ${ }^{(8)}$; en Ayacucho, con clima, geografía, epidemiología y cultura 
diferenciada y altos niveles de ruralidad, solo el $36,6 \%$ de la población tiene como lengua materna el español (25); o Puno cuya población regional se encuentra constituida básicamente por dos culturas colla y aymara, el $43 \%$ de la población tiene como lengua materna el quechua y el $33 \%$ el aymará (26). Las estrategias estipuladas en las guías nacionales, como el adecuar el lenguaje a la población y a las creencias culturales, no es una herramienta aun fortalecida en el personal de salud, pues carece de herramientas operativas para que el personal de salud las pueda ejecutar en este punto a pesar que en la norma se indique fortalecer aspectos culturales positivos y orientar con argumentos sencillos y verdaderos el por qué se deben evitar los aspectos negativos ${ }^{(21)}$.

El Sistema SIEN cuenta con una organización de personas dedicadas a dar soporte y corrección de errores de los datos registrados que, si bien solamente tiene información pasiva de registro de datos, consideramos, por la cantidad de datos y la baja frecuencia de errores encontrados $(<0,4 \%$ ), que las cifras son consistentes, por ello, el sistema SIEN podría ser considerado como una herramienta útil para la vigilancia en salud pública $(27,28)$. Si bien carece aún de conexión con otros sistemas que contienen información del estado de salud y nutrición de la población, ello hace muy difícil tratar de encontrar factores asociados al problema, además, el sistema aún no puede brindar rapidez en la respuesta de la información, pues depende de muchos actores regionales, por lo que la información no es presentada oportunamente.

Según los hallazgos del presente estudio a partir de los 13 años casi un tercio de las gestantes adolescentes presentan anemia. Sin embargo, se encuentra mucha variabilidad en las cifras porcentuales entre los 10 a 12 años, probablemente porque la muestra fue pequeña, debido a que es menos frecuente encontrar gestantes adolescentes en ese rango de edad.

El proceso de hiperhidrosis en las gestantes puede ocasionar reducción de los niveles de $\mathrm{Hb}$, según los trimestres se puede determinar que los porcentajes de anemia se incrementan conforme aumentan los trimestres de gestación, siendo para el año 2012 de 15,8\% para el primer trimestre, de $16,6 \%$ para el segundo trimestre y de $32,5 \%$ para el tercer trimestre, este comportamiento se evidenció en todos los años. El hierro y los folatos son nutrientes esenciales para la salud materno-fetal, la carencia de estos elementos en la dieta produce anemia nutricional, se manifiestan en la madre con menor capacidad de trabajo, disminución de la actividad física, mayor riesgo de parto prematuro, hemorragias, producto de bajo peso al nacer y alteraciones en el sistema nervioso central por la insuficiente mielinización y síntesis de neurotransmisores (25). Los efectos de la deficiencia de hierro durante el embarazo darán como resultado una disminución en la concentración de hemoglobina materna que, a su vez, explica un balance negativo de hierro. El tratamiento profiláctico aumenta los niveles de la hemoglobina lo cual es un efecto benéfico de la suplementación ${ }^{(26)}$.

Agradeciemientos: al equipo del Sistema de Información del Estado Nutricional (SIEN) de la Dirección Ejecutiva de Vigilancia Alimentaria y Nutricional (DEVAN) del Centro Nacional de Alimentación y Nutrición (CENAN) del Instituto Nacional de Salud (INS): Lic. Juan Carlos Barboza, Ing. Manuel Prado y Téc. Carlos Cosser. Al equipo técnico SIEN por DIRESA Amazonas: Liliana Mori. Ancash: Alida Núñez y Delia Torres. Andahuaylas: Delia Juárez, Nelyda Coaquira y Rafael Huaranga. Apurímac: Jannett Jara y Jorge Quispe. Arequipa: Ana Manco y Sandrino Rojas. Ayacucho: Alberta Choque, Enrique Pozo y Priscila Escobedo. Cajamarca: Iginia Arce. Callao: Julio García y Luz Cuya. Chota: Marisol Campomanez y Maritza Vásquez. Cusco: Nancy Meza. Cutervo: César Aldana y José Chepe. Huancavelica: Doris Idone y Rodolfo Cóndor. Huánuco: Guadalupe Huamán y Patricia Delgado. Ica: Blanca Mora y Rina Phun. Jaén: Rossana Ortiz. Junín: José Rojas y Silvia Villegas. La Libertad: Elida Márquez y Elva Gil. Lambayeque: Jorge Chancafe y Martha Ynami. Lima Ciudad: Rosa Cruz. Lima Este: Pierina Cuellar. Lima Sur: Elena Rivera. Lima: Lida Chafalote. Loreto: Ana Arroyo. Madre de Dios: Soledad Cárdenas. Moquegua: Antonieta Risco y Janett Ccopacati. Pasco: Percy Otárola. Piura: Gladys Castro y Judit Huamán. Puno: Noemí Ayma y Luz Flores. San Martín: Martha Panduro. Sullana: Carmela Núñez. Tacna: Dina Paredes y Pablo Ameri. Tumbes: Darwin Sosa y Teresa Gavidia. Ucayali: Ángel Urquia y Janet Cruz.

Contribuciones de autoría: OM participó en la concepción y diseño del artículo, análisis e interpretación de datos, redacción del artículo, revisión crítica del artículo y asesoría estadística. GG participó en la concepción y diseño del artículo, análisis e interpretación de datos, revisión crítica del artículo, aprobación de su versión final, asesoría estadística y asesoría técnica o administrativa.

Fuentes de financiamiento: los autores declaran no tener conflictos de interés.

Conflictos de interés: autofinanciado. 


\section{REFERENCIAS BIBLIOGRÁFICAS}

1. Barbón O. Algunas consideraciones sobre comunicación, género y prevención del embarazo adolescente. Rev Cubana Hig Epidemiol. 2012;50(2):245-9.

2. Alvarado R, Martínez O, Baquero M, Valencia C, Ruiz A. Problemática en torno al embarazo en adolescentes de 14 a 19 años, en Nocaima (Cundinamarca). Rev Cienc Salud Bogota (Colombia). 2007;5(1):40-52.

3. Instituto Nacional de Estadística e Informática. Perú Encuesta Demográfica y de Salud FamiliarENDES: Nacional y Departamental 2013. Lima: INEI; 2014.

4. Veloza M. Salud familiar en familias con adolescente gestante. Avances en Enfermeria. 2012;30(1):75-81.

5. Menéndez G, Navas I, Hidalgo Y, Espert J. El embarazo y sus complicaciones en la madre adolescente. Rev Cubana Obstet Ginecol. 2012;38(3):333-42.

6. Alcázar L; Grupo de Análisis para el Desarrollo, Acción contra el Hambre. Impacto económico de la anemia en el Perú. Lima: Arteta; 2012.

7. Lazarte $S$, Issé B. Prevalencia y etiología de anemia en el embarazo. Estudio observacional descriptivo en el Instituto de Maternidad de Tucumán. Rev Argent Salud Publica. 2011;2(8):2835.

8. Munares-García O, Gómez-Guizado G, Barboza-Del Carpio J, SánchezAbanto J. Niveles de hemoglobina en gestantes atendidas en establecimientos del Ministerio de Salud del Perú, 2011. Rev Peru Med Exp Salud Pública. 2012;29(3):329-36.

9. Ministerio de Salud, Instituto Nacional de Salud. Guía Técnica: Procedimiento parala determinación dela hemoglobina mediante hemoglobinómetro portátil. Lima: MINSA; 2013.

10. Méndez R, Pacheco B, Noriega $H$, Quihui L, Morales G, Valencia M. Prevalencia de deficiencia de hierro y de anemia por deficiencia de hierro en adolescentes embarazadas del nordeste de México 2007-2008. Arch Latinoam Nutr. 2009;59(2):147-151.

11. Organización Mundial de la Salud. Concentraciones de hemoglobina para diagnosticar la anemia y evaluar su gravedad. Ginebra: OMS; 2011.

12. Barba-Oropeza F, Cabanillas-Gurrola J. Factores asociados a la anemia durante el embarazo en un grupo de gestantes mexicanas. Arch Med Fam. 2007;9(4):170-5

13. Ortega P, Leal J, Chávez C, Mejías L, Chirinos N, Escalona C. Anemia y depleción de las reservas de hierro en adolescentes gestantes de una zona urbana y rural del estado Zulia, Venezuela. Rev Chil Nutr. 2012 Sep;39(3):11-7.

14. Quintero R, Muñoz N, Álvarez L, Medina G. Estado nutrional y seguridad alimentaria en gestantes adolescentes. Pereira, Colombia, 2009. Revista Investigación y Educación en Enfermería. 2010 Jul;28(2):204-13.

15. Rodríguez O, Fernández S, Gazapo R, Fernández E, Rodríguez T, Sánchez R, et al. Factores que inciden en la anemia ferropénica de la embarazada. Rev Cubana Fam. 2002;36(3):176-81.

16. Perú, Ministerio de Economía y Finanzas. Informe de cumplimiento de metas de indicadores priorizados del convenio de apoyo presupuestario al programa articulado nutricional - EUROPAN Tramo variable año 2011. Lima: Ministerio de Economía y Finanzas; 2012.

17. Perú, Ministerio de Salud, Unicef. Estudio de adherencia a la suplementación con hierro durante la gestación en las direcciones de salud de Apurímac y Ayacucho. Lima: MINSA; 2009.

18. Breymann C. Tratamiento de la anemia por deficiencia de hierro en el embarazo y el posparto. Rev Peru Ginecol Obstet. 2012;58(4):313-28.

19. Haider BA, Bhutta ZA. Suplementos de múltiples micronutrientes para mujeres durante el embarazo. Base de Datos Cochrane de Revisiones Sistemáticas. 2007;4:CD004905. doi: 10.1002/14651858.CD004905.pub2.

20. Instituto Nacional de Salud. Consejería nutricional en el marco de la atención de salud materno infanti: documento técnico. Lima: MINSA; 2010.

21. Perú, Ministerio de Salud. Guías Nacionales de Atención Integral de la Salud Sexual y Reproductiva. Lima: MINSA; 2004.

22. Ailinger R, Moore J, Pawloski L, Zamora L. Conceptos de anemia entre mujeres nicaragüenses de baja renta. Rev Lat-Am Enferm. 2009;17(2):1-6.

23. Sobrevilla D. La filosofía andina del P. Josef Estermann. Quito: Abya-Yala, 1998;359 pp. Solar. 2008;4(4):231-47.

24. Nureña C. Incorporación del enfoque intercultural en el sistema de salud peruano: la atención de parto vertical. Rev Panam Salud Publ. 2009;26(4):368-76.

25. Perú, Ministerio de Salud, Dirección Regional de Salud Ayacucho. Análisis de la Situación de salud de Ayacucho 2011. Ayacucho: MINSA; 2012.

26. Perú, Ministerio de Salud, Dirección Regional de Salud Puno. Análisis de la situación de salud de la región Puno 2010. Puno: MINSA; 2010.

27. Martínez J. De la información a la acción: la vigilancia de la salud pública. Rev Esp Salud Public. 2000;74:81-5.

28. Bardasquera D. La vigilancia en salud. Elementos básicos que debe conocer el médico de familia. Rev Cubana Med Gen Integr. 2002;18(1):76-81.

Correspondencia: Oscar Munares García Dirección: Av. Tizón y Bueno 276 Jesús María

Teléfono: 6176200 Anexo 6605

Correo electrónico:omunares@ins.gob.pe 